\title{
Die Präsidentin berichtet
}

Gabriela Rüttimann

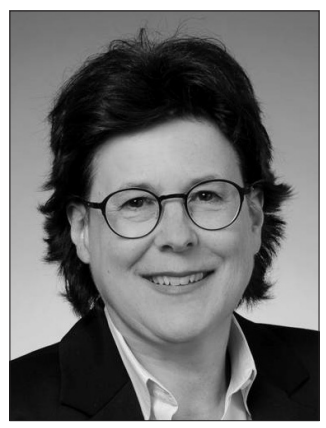

Spätsommer-Klausurtagung des Vorstands

Zum Abschluss des Sommers fand am schönen Vierwaldstättersee die jährliche Retraite unseres Vorstandes statt. Die reich befrachtete Traktandenliste reichte von A wie Anordnungsmodell bis $Z$ wie Zukunftsvision für die ASP. Hier wurde die zu Beginn des Jahres eingeleitete Strategiediskussion zu Ende geführt und ein Massnahmenpaket beschlossen. Die Strategie 2019-2022 wird im Frühling 2019 der Mitgliederversammlung vorgestellt.

Die Vorbereitungen zum 40-Jahr-Jubiläum der ASP sind in vollem Gange. Die Jubiläumsfeier mit vorgängiger Mitgliederversammlung findet in Zürich im KOSMOS statt. In einer Jubiläumsbroschüre wird die Geschichte des Verbands und damit die Entwicklung der Psychotherapie in der Schweiz von dem Historiker Walter Aeschimann aufgearbeitet. Wie bereits angekündigt, ist der Anlass für den 23. März 2019 geplant. Nach der Mitgliederversammlung findet die Jubiläumsveranstaltung mit anschliessendem Abendessen statt. Das Programm und die Einladungen werden rechtzeitig verschickt.

\section{Rechtliche Schritte beschlossen}

Bis zum Redaktionsschluss war nicht klar, ob wir in Sachen Anordnungsmodell einen Schritt weiterkommen würden. Beunruhigend war allemal, dass auf oberster politischer Ebene keine klare

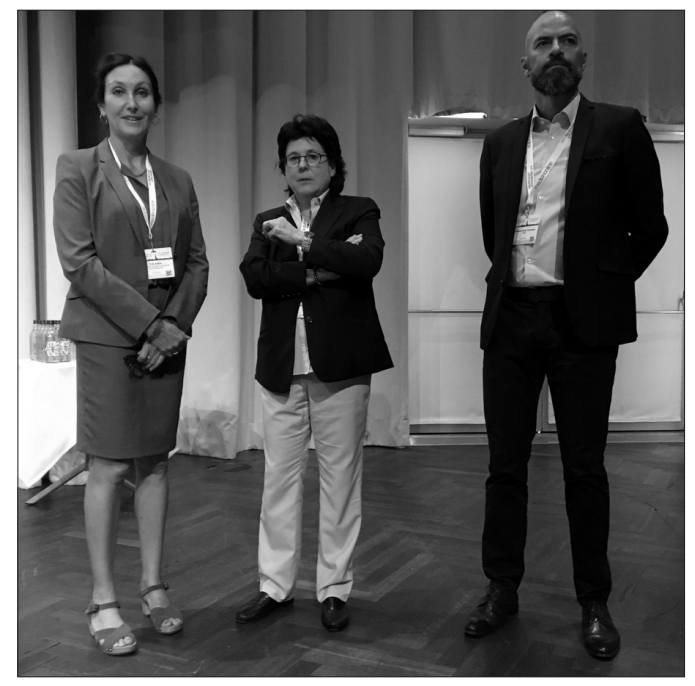

Die Präsident/innen der Verbände: (v. I.)

Yvik Adler (FSP), Gabriela Rüttimann (ASP),

Christoph Adrian Schneider (SBAP)
Unterscheidung zwischen Anordnungs- und Delegationsmodell gemacht wurde und sich das Interesse an unserem Anliegen in Grenzen zu halten scheint. Was immer geschehen wird, ist die feste Absicht der ASP, ein Rechtsverfahren auf den Weg zu bringen mit dem Ziel, die Abrechnung von Psychotherapiebehandlungen ohne Delegation mit der Grundversicherung zu ermöglichen. Dass ein solches Verfahren einen langen Atem braucht, ist uns bewusst. Wir sind jedoch nicht länger bereit, diese Hinhaltetaktik Jahr um Jahr hinzunehmen. Daran ändert auch der Antwortbrief von Bundesrat Alain Berset nichts, der einmal mehr in Aussicht stellte, dass das Verfahren unter Einbezug der involvierten Verbände weitergehen würde.

\section{Wieviel klinische Praxis ist «genügend»?}

Das Psychologieberufegesetz PsyG verlangt, dass Studierende in ihrer Weiterbildung «genügend» klinische Praxis vorweisen müssen. Die Ausgestaltung der klinischen Praxis überlässt es den verantwortlichen Organisationen. Da die ASP die Verantwortung für die Institute übernommen hat, die sich für ihre Akkreditierung dem Konzept ASP Integral angeschlossen haben, ist es an uns festzulegen, wie viel «genügend» ist. Diesen Punkt gilt es ebenfalls mit den Kollektivmitgliedern zu besprechen, die in der Charta-Konferenz zusammengeschlossen sind. Dies ist eine Gelegenheit, sie für das Thema zu sensibilisieren. Bezüglich Studierenden, die einen Teil ihrer Weiterbildung im Ausland absolviert haben, muss ebenfalls eine Regelung gefunden werden. Anlässlich eines konkreten Falles im Kanton Tessin legte die ASP fest, dass von den zwei vorgeschriebenen Jahren klinischer Praxis mindestens ein halbes Jahr in einer Einrichtung der psychotherapeutisch-psychiatrischen Versorgung in der Schweiz absolviert werden muss. Diese Tätigkeit soll der Kandidatin oder dem Kandidaten gleichzeitig ermöglichen, das Schweizer Gesundheitswesen von innen kennenzulernen. Die Supervision muss zu 100 Prozent in einem akkreditierten Schweizer Weiterbildungsinstitut absolviert werden.

\section{Erfolgreicher Psy-Kongress 2018}

Als Kooperationspartnerin der Schweizerischen Gesellschaft für Psychiatrie und Psychotherapie (SGPP) nahm die ASP wie bereits vor vier Jahren am diesjährigen Psy-Kongress teil, der im 
September während drei Tagen im Kursaal Bern stattfand. Der Kongress wurde unter dem Titel «Bausteine in der Behandlung und Betreuung psychisch kranker Menschen» durchgeführt. Die rund 1.200 Teilnehmerinnen und Teilnehmer hatten die Gelegenheit, an zahlreichen Symposien, Kursen und Open Space-Veranstaltungen teilzunehmen, die ein sehr breites Spektrum an Themen über Psychiatrie und Psychotherapie abdeckten.

Die drei Verbände steuerten ein Panel bei zum Thema «Versorgung psychisch Kranker in der Schweiz: Heute und in der Zukunft». Der Diskurs vonseiten der Gesundheitspolitiker lässt nichts Gutes erahnen und bleibt in der Ausgabeneutralität stecken. Die Aussagen der Behördenvertreter waren unbestimmt oder drehten sich im Kreis. Die Vertreterinnen und Vertreter des Bundesamts für Gesundheit (BAG) und der Santésuisse versteckten sich hinter Gesetzen, sodass der Eindruck entstand, dass sich auch in den nächsten zehn Jahren nichts verändern wird. Die Überalterung und der fehlende Nachwuchs in der Psychiatrie rufen geradezu nach einer Gleichstellung des Psychotherapieberufes. Es scheint jedoch, dass das BAG die Lösung der Überalterung im Engagement von Psychiaterinnen und Psychiatern aus dem Ausland sieht, was die lakonische Antwort eines Panelteilnehmers auslöste, dass man bald einen Sprachkurs machen müsse, wenn man in ein Spital eintrete.

Die ASP arbeitete im Organisationskomitee des Kongresses mit und stellte ein Mitglied des Wissenschaftskomitees, das einerseits die Themen der verschiedenen Veranstaltungen setzte und andererseits die Abstracts sichtete. Die ASP-Geschäftsleiterin betätigte sich als Mitglied in der Poster-Jury. Zudem vermittelten wir mit Rosmarie Barwinski ein ASP-Mitglied zur Durchführung eines Workshops. Die sehr gut besuchte und spannende Veranstaltung zum Thema «Resilienz fördern in der Traumatherapie» illustrierte schrittweise, wie die Aufarbeitung eines Traumas während der Psychotherapiebehandlung die Widerstandsfähigkeit der Patientin oder des Patienten steigern kann.

\section{Neues Vorstandsmitglied der Suisse Romande in Aussicht}

Die ASP ist sehr erfreut, mit Sandra Feroleto für die Suisse Romande ein neues Vorstands-

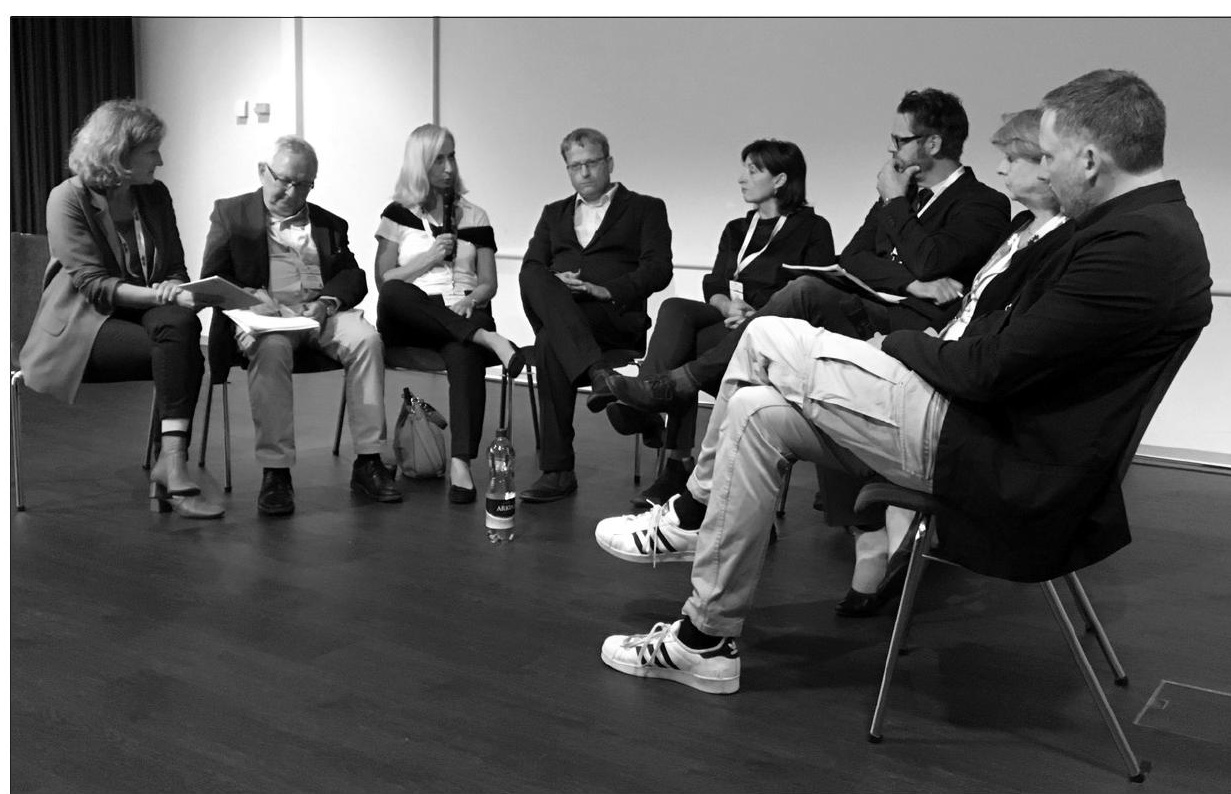

Am Panel beteiligten sich (v. I.) Sabine Schläppi, Sämi Rom, Dr. Magdalena Berkhoff, Daniel Habegger, Dr. Fulvia Rota, Stefan Spycher, Erika Ziltener, Simon Hofer.

mitglied präsentieren zu können, das sie der Mitgliederversammlung zur Bestätigungswahl vorschlagen wird. Damit ist der Vorstand wieder komplett und die Vertretung in allen Sprachregionen gewährleistet. Nach Meinung des Vorstands ist sie eine sehr geeignete Kandidatin und würde ausgezeichnet in das bestehende Team passen.

Sandra Feroleto ist Direktorin der Assoziation La Branche in Savigny mit 340 Mitarbeitenden, die 140 Kinder und Erwachsene mit einer psychischen Behinderung in ihrem Schulunterricht, der Bildung, Arbeit und in ihrem Leben begleiten. Daneben ist sie selbständige Psychotherapeutin in ihrer Praxis Artepsy in St-Prex. Ihre Weiterbildung in Psychotherapie hat sie an der Ecole Française d'Analyse Psycho-Organique (EFAPO) in Paris absolviert. Die Ausund Weiterbildung hat sie mit verschiedenen Kursen an Fachhochschulen und Universitäten ergänzt, wie z.B. mit einem CAS in Mediation und EMDR-Praxis für Kinder und Erwachsene. Als Mitglied der ASP und als gelegentliche Delegierte der EFAPO in der Charta hat sie bereits Kontakt zum Verband gepflegt, sodass ihr unsere Haltung und berufliche Ausrichtung bekannt sind.

Gabriela Rüttimann ist Präsidentin der ASP. 